Check for updates

Cite this: RSC Adv., 2018, 8, 37835

Received 20th September 2018 Accepted 26th October 2018

DOI: $10.1039 / c 8 r a 07838 f$

rsc.li/rsc-advances

\section{Micro-flow nanocatalysis: synergic effect of TfOHaSPIONs and micro-flow technology as an efficient and robust catalytic system for the synthesis of plasticizers $\dagger$}

\author{
Maryam Tashi, ${ }^{a}$ Behnaz Shafiee, ${ }^{a}$ Yoshie Sakamaki, ${ }^{b}$ Ji-Yun Hu, (DD ${ }^{b}$ Zachary Heidrick, $^{b}$ \\ Ahmad R. Khosropour ${ }^{\star a b}$ and M. Hassan Beyzavi (D) *b
}

\begin{abstract}
The combination of continuous flow technology with immobilizing of only 0.13 mol\% of triflic acid (TfOH) on silica-encapsulated superparamagnetic iron oxide nanoparticles (SPIONs) under solvent-free conditions successfully provided a powerful, efficient, and eco-friendly route for the synthesis of plasticizers. The turnover frequency value in micro-flow conditions varied in the range of 948.7 to $7384.6 \mathrm{~h}^{-1}$ compared to 403.8 to $3099 \mathrm{~h}^{-1}$ for in-flask. This technique works efficiently, encouraging future applications of micro-flow nano-catalysis in green chemistry.
\end{abstract}

The investigation of powerful and less hazardous chemical catalysts has become a priority in developing sustainable organic transformations., ${ }^{1,2}$ Triflic acid (TfOH), as a versatile Brønsted acid catalyst, has been used widespread in organic transformations. ${ }^{3-11}$ However, the difficulties in handling and transportation of $\mathrm{TfOH}$ associated with its highly corrosive nature, restrict its industrial applications. ${ }^{12}$ Moreover, the recovery of $\mathrm{TfOH}$ from the reaction mixture leads to the formation of large amounts of waste. ${ }^{12,13}$ To solve these problems, immobilization of $\mathrm{TfOH}$ onto an inorganic matrix as a heterogeneous catalyst is an alternative, which features easyhandling, low toxicity, superior stability, etc. Despite these advantages, the obtained catalyst still suffers several drawbacks, such as low surface area, the monotonous recycling by filtration, and loss of catalyst during recovery. ${ }^{14-18}$ Recently, a new TfOH catalyst based on silica-coated magnetic nanoparticles was reported by Zhang and co-workers and showed high efficiency, good recyclability, and reusability in the transformation of aldehydes or ketones into their corresponding gem-dihydroperoxides. ${ }^{19}$ Therefore, extension of investigation on this superacid generation, which could produce more fine chemicals and further increase the efficiency via combining new technology, is in high demand. Nowadays, there is a growing interest in developing of micro-flow technology for various organic transformations. ${ }^{20-24}$ This technology allows a directed

${ }^{a}$ Department of Chemistry, University of Isfahan, 81746-73441, Isfahan, Iran. E-mail: arkhosropour@chem.ui.ac.ir

${ }^{b}$ Department of Chemistry and Biochemistry, University of Arkansas, Fayetteville, Arkansas 72701, USA. E-mail: beyzavi@uark.edu

$\dagger$ Electronic supplementary information (ESI) available. See DOI: $10.1039 / \mathrm{c} 8 \mathrm{ra} 07838 \mathrm{f}$ reactant flux through an immobilized catalyst, making it attractive by enhancing mass- and heat-transfer characteristics via low hold-up volumes. Micro-flow technology has also an excellent thermal transport capability, as well as the ability to efficiently optimize reaction conditions by controlling the residence time..$^{25-28}$ Moreover, the yields of reactions performed in the microreactor at a very short residence time, even for scaled up reactions, are much higher than those obtained via conventional stirring..$^{29}$ Therefore, integrating the advances of micro-flow chemistry into the $\mathrm{TfOH}$ heterogeneous catalyst would bring new features, such as higher yields and efficient optimization, in addition to the good recyclability and reusability. Micro-flow nanocatalysts could serve as a new and practical technique for overcoming of the problems mentioned above.

To this end, we prepared $\mathrm{TfOH}$ functionalized silicaencapsulated superparamagnetic iron oxide nanoparticles (TfOH@SPIONs) and tested their efficiency in synthesizing plasticizers with micro-flow techniques. Plasticizers are an essential ingredient for the producing of macromolecule materials used widely by polymer industries. ${ }^{30,31} \mathrm{~A}$ variety of modified catalytic processes have been performed; from traditional catalysts, such as sulfuric acid ${ }^{32}$ and titanate, ${ }^{33}$ to more modern ionic liquids ${ }^{34}$ for the synthesis of plasticizer esters. Although these methods are valuable, most of them require hours of reflux, utilizing expensive or corrosive catalysts, tedious work-up, difficult recovery, and easy deactivation with consumption of energy. Therefore, implementation of ecofriendly and straightforward chemical methods remains a particularly interesting task.

The aforementioned explanations and our recent investigations on the micro-flow nano-catalyst technology, ${ }^{35}$ encouraged 
us to develop the ultimate green synthesis, very practical, and straightforward protocol for the synthesis of plasticizer esters via the first combination of TfOH@SPION with micro-flow technique.

\section{Result and discussion}

The TfOH@SPION was characterized by Fourier transform infrared spectroscopy (FT-IR), powder X-ray diffraction (XRD), transmission electron microscopy (TEM), and vibrating sample magnetometry (VSM).

The FT-IR spectrum of TfOH@SPION shows absorption bands at $3411 \mathrm{~cm}^{-1}$ (O-H stretching vibration), 1377, 1178, $1040 \mathrm{~cm}^{-1}\left(\mathrm{O}=\mathrm{S}=\mathrm{O}\right.$ and $\mathrm{SO}_{3}{ }^{-}$stretching bands $), 646 \mathrm{~cm}^{-1}(\mathrm{Si}-$ O), and $635-587 \mathrm{~cm}^{-1}(\mathrm{Fe}-\mathrm{O})$, respectively (Fig. S1, ESI $\dagger$ ). The XRD spectrum of the TfOH@SPION was similar to that of SPION which illustrated that no structural changes occurred during immobilization of TfOH (Fig. S2 $\dagger$ ). TEM images of TfOH@SPION revealed that it appears to have almost a cubic structure with the average size about 13-15 nm (Fig. S3†). The dc magnetic characterization of $\mathrm{Fe}_{3} \mathrm{O}_{4}$, the neat SPION nanoparticles, and TfOH@SPION were examined at room temperature in an external field range of $\pm 10 \mathrm{kOe}$. In this investigation, due to the functionalization of SPIONs by TfOH, Ms (saturation magnetization) was found to be $10 \mathrm{emu} \mathrm{g}^{-1}$, which is considerably lower than $40 \mathrm{emu} \mathrm{g}^{-1}$ of the SPIONs (Fig. S4 $\dagger$ ).

After structural characterization of the catalyst, the catalytic activity of TfOH@SPION in the preliminary studies was evaluated through batch manner for the synthesis of a general commercial plasticizer, diisobutylphthalate (DIBP), as a model. For comparison, six frequently used catalysts as well as TfOH@SPION, were investigated under the same conditions. The template reaction was first run with stirring of phthalic anhydride $(1 \mathrm{mmol})$ and 2 -isobutanol $(2 \mathrm{mmol})$ in the presence of $0.13 \mathrm{~mol} \%$ various catalysts (based on active sites) at $100{ }^{\circ} \mathrm{C}$ for $200 \mathrm{~min}$. As shown in Fig. 1, TfOH@SPION gave the highest yield $(70 \%)$ among all the catalysts investigated. $p$-Toluenesulfonic acid ( $p$-TSA, 50\%) and Amberlyst 15 (35\%) showed moderate yields, while $n$-butyltin dihydroxychloride, pentadecafluorooctanoic acid, Nafion-H and trichlorotriazine did not catalyze the reaction.

Next, the TfOH loading amount of TfOH@SPION was examined (Fig. 2). The yield of DIBP increases with higher

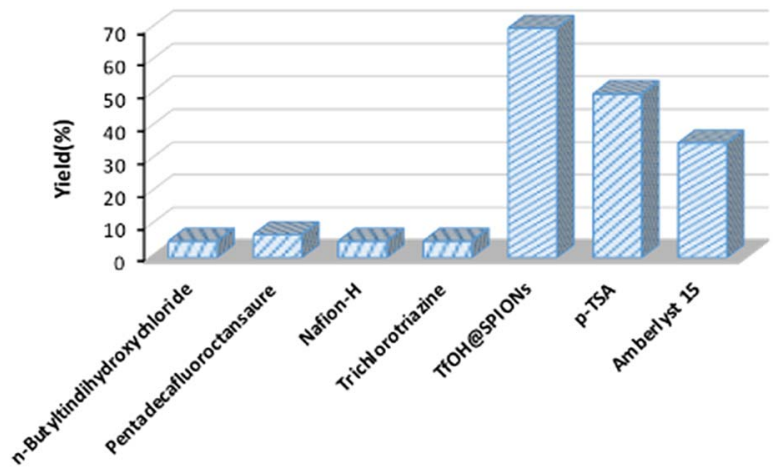

Fig. 1 Assessment of different catalysts on the model reaction.

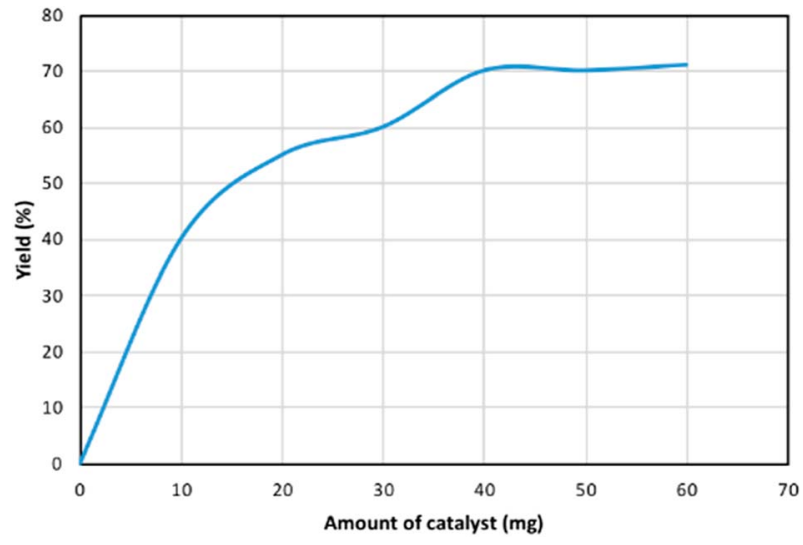

Fig. $2 \mathrm{TfOH}$ loading effect of TfOHaSPIONs for the reaction of phthalic acid $(1.0 \mathrm{mmol})$ and 2-butanol $(2.0 \mathrm{mmol})$ at $100{ }^{\circ} \mathrm{C}$ after $200 \mathrm{~min}$.

loading TfOH of TfOH@SPION, which comes to the plateau of about $70 \%$ yield at a loading level of $40 \mathrm{mg}$ (corresponds to $0.13 \mathrm{~mol} \%$ ). Notably, lower yields were obtained when the same reaction was carried out with lower amount of the catalyst (Fig. 2). Moreover, no reaction occurred in the absence of a catalyst and no valuable yield was obtained in the presence of SPION without loading of TfOH.

Given these promising results, we next tried to optimize the reaction conditions by changing the method from batch to continuous flow technology. Based on the optimized batch conditions, the effect of reaction temperature and flow rate on the reaction efficiency were further studied.

The condition for the initial examinations, particularly the catalyst amount and substrate molar ratio, were selected according to our results obtained for the batch method. An initial study revealed that the residence time and the reaction temperature were crucial factors for this transformation. Periodic analyses of the reaction mixture illustrated the effect of the residence time ( $t: 0.5,1,1.5$ and $2 \mathrm{~h}$ ) and temperature $(T: 70,80$, 90 and $100{ }^{\circ} \mathrm{C}$ ) to the yield of the product (Fig. 3).

As shown in Fig. 3, combinatorial sixteen conditions from four temperatures $\left(70,80,90,100^{\circ} \mathrm{C}\right)$ and four residence time $(0.5,1.0,1.5,2.0 \mathrm{~h})$ were systematically examined. Generally, either increasing temperature, or prolonging residence time could increase the product yield. For example, at $70^{\circ} \mathrm{C}$, the yield

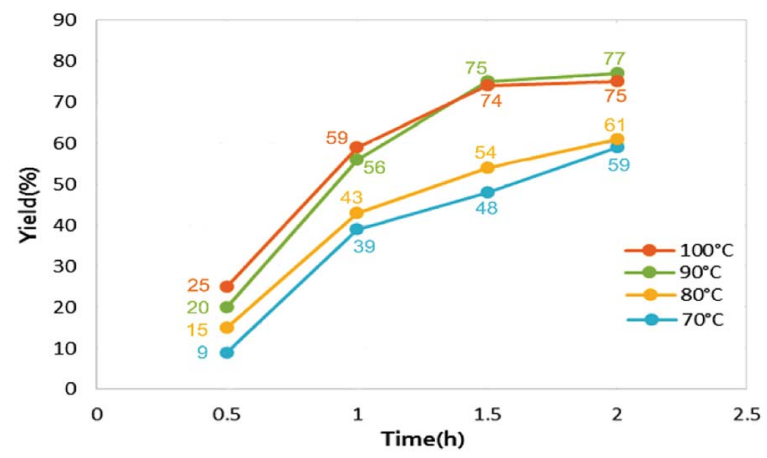

Fig. 3 Examination of temperature and residence time effects in the micro-flow technique. 
Table 1 Screening of the ester plasticizer synthesis catalyzed by TfOH@SPIONs

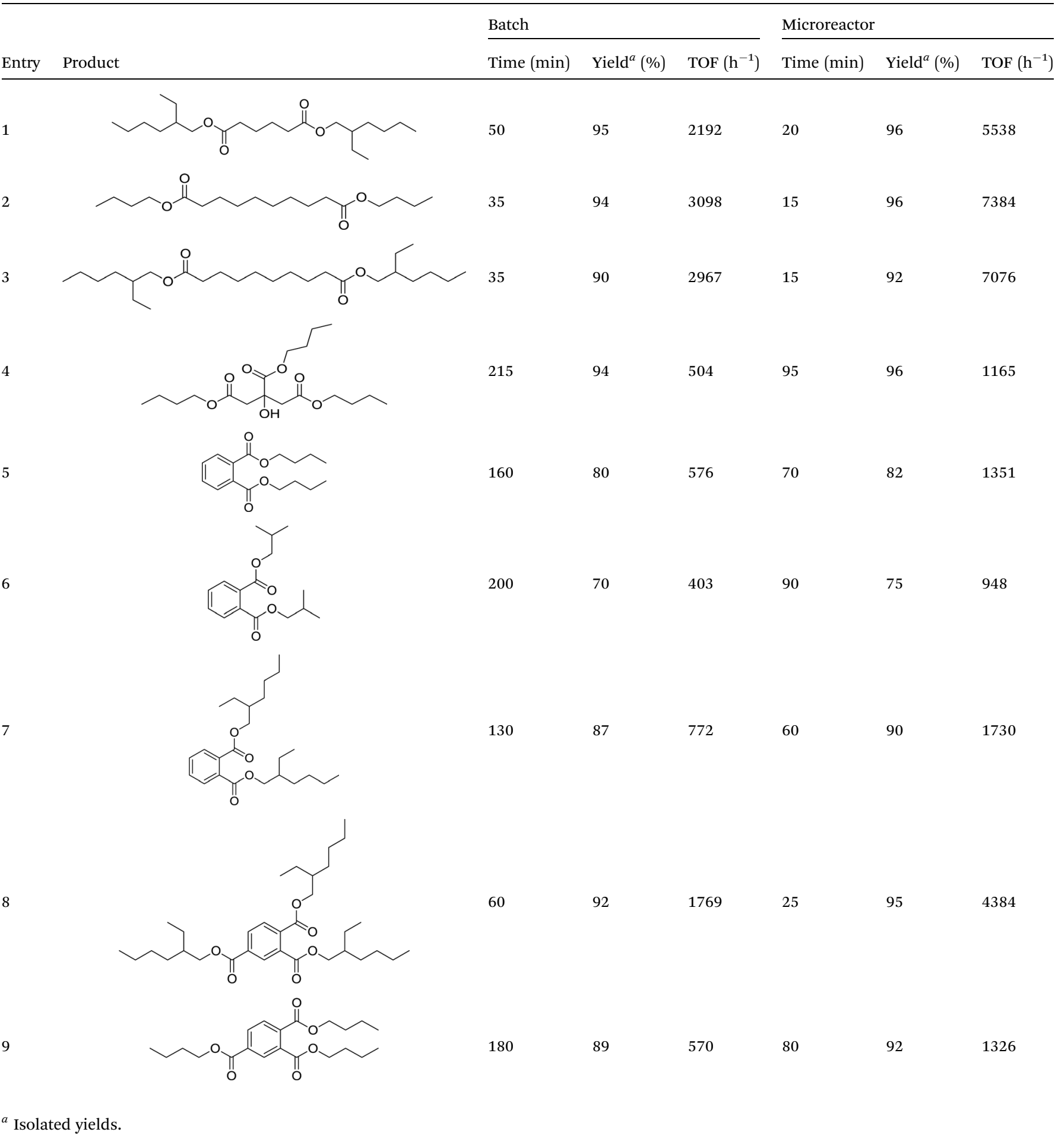

was increased from 9 to $39,48,59 \%$ respectively with the residence time extended from 0.5 to 1.0 , between 1.5 to $2.0 \mathrm{~h}$, respectively. Similarly, keeping the same residence time of $0.5 \mathrm{~h}$, the product yield was increased from 9 to $15,20,25 \%$ when temperature was elevated from 70 to 80 , to 90 , to $100{ }^{\circ} \mathrm{C}$, respectively. These effects approach the maximum at $90{ }^{\circ} \mathrm{C}$ with residence time of $1.5 \mathrm{~h}$, giving a yield of $74 \%$, where further increase the temperature or the residence time didn't improve the yield significantly (only 1\% higher). Therefore, a reaction temperature of $90{ }^{\circ} \mathrm{C}$ and a residence time of $1.5 \mathrm{~h}$ (corresponds to a flow rate of $0.4 \mathrm{~mL} \mathrm{~h}^{-1}$ in this study) were used as the optimal conditions for the following studies. Next, we evaluated the scope and generality of this procedure and the results are summarized in Table 1.

As shown in Table 1, the TfOH@SPION catalyst shows a broad substrate scope with yields ranging between 70-96\%. In 


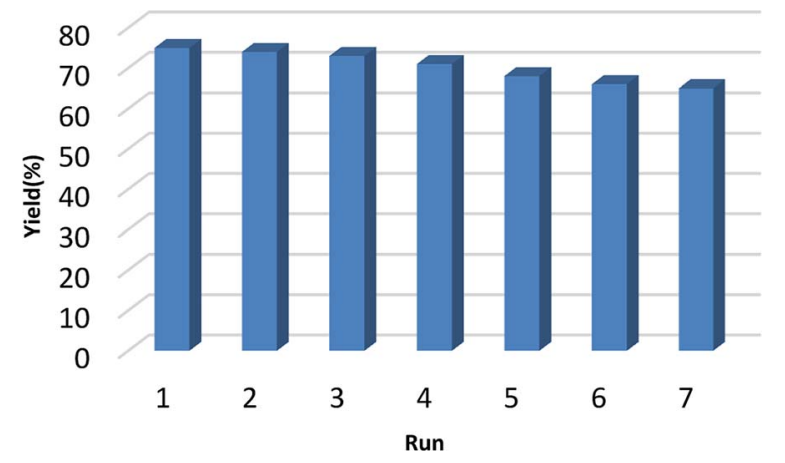

Fig. 4 Reusability of TfOH@SPIONs for the synthesis of DIBP under flow technology.

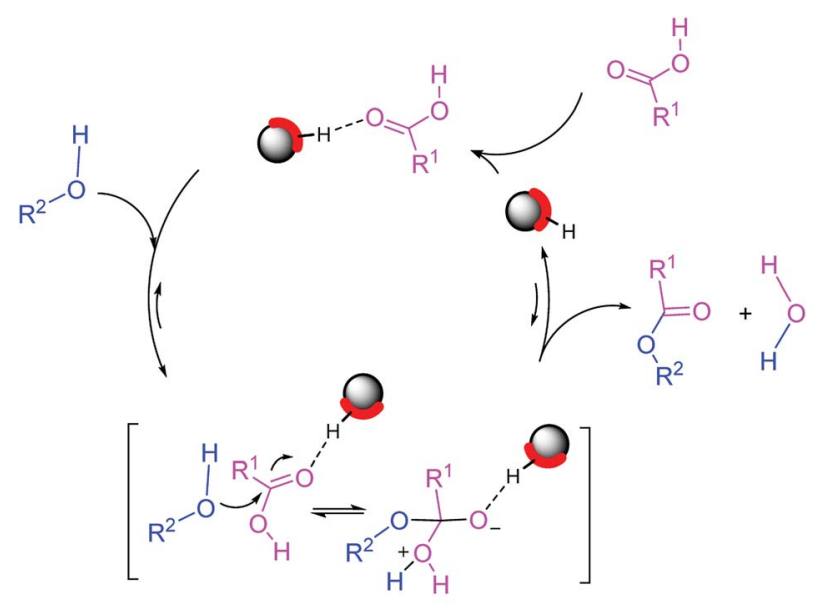

$Q_{H}:$ TfOH@SPIONs

Fig. 5 Plausible mechanism.

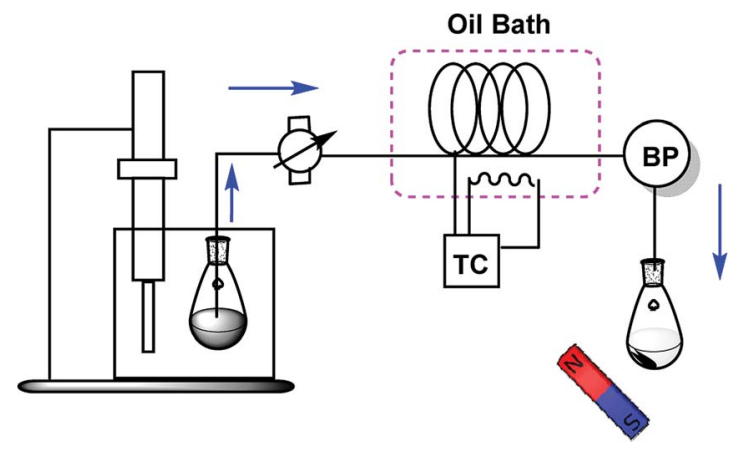

Scheme 1 Microflow setup for the preparation of plasticizers.

all cases, micro-flow technology-assisted reactions were superior to the traditional batch reactions in terms of yield and reaction time. The reaction time of microreactor was generally shortened more than half of that of the batch reactions, while still giving a slightly higher yield. The turnover frequencies (TOF) in micro-flow technology is $948.7-7384.6 \mathrm{~h}^{-1}$ compared to 403.8-3099 $\mathrm{h}^{-1}$ in traditional manner, which may be attributed to better dispersion of the nano-catalyst in a minimum volume in contrast to magnetically stirred reactions. Moreover, this protocol avoids the problems associated with catalyst handling, safety, and pollution. To the best of our knowledge, this is the first example of efficient synthesis of plasticizers using the combination of nano-catalyst with micro-flow technology.

It is noteworthy to mention that the reusability and recyclability of a catalyst has the positive effect on diminishing the waste and reducing of the production cost. In this study, TfOH@SPIONs could be conveniently recovered by applying an external magnetic field and the recovered catalyst were reused for at least six times without any substantial loss of its catalytic performance. As demonstrated in Fig. 4, the product yield of the seventh run for the synthesis of DIBP under flow chemistry, is $65 \%$ only slightly lower than $75 \%$ of the first run.

Finally, encouraged by the above results, the scale-up synthesis of DIBP was also investigated as the model reaction. We increased the scale of the reaction to $5 \mathrm{mmol}$, keeping the reaction stoichiometry intact. The reaction proceeded as expected and the product was obtained with similar yields $(75 \%)$ to the small-scale reaction.

It is widely assumed that the reaction occurs via the esterification of carboxylic acid derivatives via activation of carbonyl group with acidic hydrogens on the nanoparticle surface (Fig. 5).

\section{Conclusions}

We demonstrated here a sustainable, straightforward, efficient, and continuous-flow approach to the synthesis of ester plasticizers catalyzed by TfOH@SPIONs as a practical nano-catalyst. This approach features a broad substrate scope and also could be scaled up to $5 \mathrm{mmol}$ scale. In addition, the nano-catalyst displayed elevated catalytic activity in the microreactor compared to the batch reactions, which might benefit from the synergic effect of the high specific surface area, and high mass and energy transport efficiency with micro flow technology. Furthermore, the nano-catalyst possessed high stability and could be reused for six runs without significant loss of activity.

\section{Experimental}

All chemicals were purchased from Merck Company. Silicacoated magnetite nanoparticles (SPIONs) were synthesized according to the literature. ${ }^{36}$ All known organic products were identified by the comparison of their physical and spectral data with those of authentic samples.

The catalyst was analyzed by FT-IR (Fourier transform infrared spectroscopy, Nicolet-Impact 400D spectrophotometer in $\mathrm{KBr}$ pellets and reported in $\mathrm{cm}^{-1}$ ), XRD (Powder X-ray diffraction, D8 Advance DVANCE with Davincl, Bruker), TEM (Philips CM30 unit operated at $150 \mathrm{kV}$ ) and VSM (vibrating sample magnetometer at Meghnatis Daghigh Kavir Co.).

Thin layer chromatography (TLC) was performed on UVactive aluminum-backed plates of silica gel (TLC Silica gel 60 F254). ${ }^{1}$ HNMR spectra were measured on a Bruker DPX 400 $\mathrm{MHz}$ spectrometer in $\mathrm{CDCl}_{3}$ with chemical shift $(\delta)$ given 
in ppm and coupling constants given in Hz. The sonication was performed in a UP 400S ultrasonic processor equipped with a $3 \mathrm{~mm}$ wide and $140 \mathrm{~mm}$ long probe.

\section{Catalyst synthesis}

To a mixture of SPIONs $(1.0 \mathrm{~g})$ in diethyl ether $(50 \mathrm{~mL})$, TfOH $(1.5 \mathrm{mmol})$ was added dropwise, and the resulting suspension was sonicated in the ultrasonic bath for $60 \mathrm{~min}$ at room temperature. The solvent was removed and the residue was heated under vacuum at $70{ }^{\circ} \mathrm{C}$ for $24 \mathrm{~h}$ to obtain TfOH@SPION.

\section{Microreactor designing}

The microreactor system used in this study includes an in-housemade tubing glass reactor which can be implemented in any chemistry laboratory. The microtube reactor was fabricated in a glass column (internal diameter, $0.5 \mathrm{~mm}$; internal volume, 0.6 $\mathrm{mL}$; length, $200 \mathrm{~cm}$ ), which was placed in an oil bath. The dispersed mixture of substrates and the catalyst was delivered to the microreactor by a peristaltic pump. A temperature controller (TC) was used for balancing and setting up heating and a backpressure regulator (BPR) for pressure control (Scheme 1).

\section{General procedure for synthesis of plasticizers}

A sonicated mixture of $1 \mathrm{~mL}$ dicarboxylic acid : alcohol or tricarboxylic acid : alcohol (1:2 and 1:3 molar ratio, respectively), and the catalyst $(40 \mathrm{mg}, 0.13 \mathrm{~mol} \%$ based on $\mathrm{TfOH}$ ) at ambient temperature and pressure was fed to the microreactor by a peristaltic pump. Afterwards, the flow rate of the mixture was adjusted to the desired value $\left(0.4 \mathrm{~mL} \mathrm{~h}^{-1}\right)$. The reactor was then heated at $90{ }^{\circ} \mathrm{C}$. After the residence time was reached (15-95 min), the discharge was collected out of BPR in a glass vessel equipped with an external permanent magnet (Scheme 1). The collected catalyst by the magnet, was washed two times with absolute ethanol $(2 \times 1$ $\mathrm{mL}$ ), air-dried, and used directly for the next round. After separation of the catalyst from the resulting crude product, the volatiles were removed in vacuum. The organic residue was extracted with ethyl acetate/water $(3 \times 5 \mathrm{~mL})$, and the combined organic phase was dried over anhydrous $\mathrm{Na}_{2} \mathrm{SO}_{4}$. Pure products were obtained in $75-96 \%$ yields after removal of solvent under reduced pressure.

\section{Conflicts of interest}

There are no conflicts to declare.

\section{Acknowledgements}

M. H. B. gratefully acknowledges the financial support through the startup funds from the University of Arkansas. A. R. K. gratefully thanks the Center of Excellence of Chemistry and the Research Council of the University of Isfahan (CECUI) for financial support of this work.

\section{Notes and references}

1 R. Sheldon, Green Chemistry and Catalysis, John Wiley \& Sons, 2015.
2 T. P. Umile, Catalysis for Sustainability, CRC Press, 2015.

3 R. D. Howells and J. D. Mc Cown, Chem. Rev., 1977, 77, 69-92.

4 N. C. Marziano, L. Ronchin, C. Tortato, A. Zingales and A. A. Sheikh-Osman, J. Mol. Catal. A: Chem., 2001, 174, 265-277.

5 D.-S. Wang and Y.-G. Zhou, Tetrahedron Lett., 2010, 51, 30143017.

6 N. Gigant and I. Gillaizeau, Org. Lett., 2012, 14, 4622-4625.

7 H. Ren, G. Zhao, S. Zhang, P. Cui and J. Huang, Catal. Commun., 2012, 18, 85.

8 S. R. Mothe and P. W. H. Chan, J. Org. Chem., 2009, 74, 58875893.

9 T. Jin, M. Himuro and Y. Yamamoto, Angew. Chem., Int. Ed., 2009, 48, 5893-5896.

10 M. Blocker, S. Immaneni and A. Shaikh, Tetrahedron Lett., 2014, 55, 5572-5575.

11 Z. P. Tachrim, L. Wang, Y. Murai, T. Yoshida, N. Kurokawa, F. Ohashi, Y. Hashidoko and M. Hashimoto, Catalysts, 2017, 7, 40-68.

12 W.-Sh. Wang, P. Chen and Y. Tang, Tetrahedron, 2017, 73, 2731-2739.

13 A. S. Jadhav and R. V. Anand, Eur. J. Org. Chem., 2017, 37163721.

14 M. Chidambaram, D. Curulla-Ferre, A. P. Singh and B. G. Anderson, J. Catal., 2003, 220, 442-456.

15 D. O. Bennardi, G. P. Romanelli, J. C. Autino and L. R. Pizzio, Appl. Catal., A, 2007, 324, 62-68.

16 D. O. Bennardi, G. P. Romanelli, J. C. Autino and L. R. Pizzio, Catal. Commun., 2009, 10, 576-581.

17 P. N. Liu, F. Xia, Q. W. Wang, Y. J. Ren and J. Q. Chen, Green Chem., 2010, 12, 1049-1055.

18 S. Khaksar, M. Tajbakhsh and M. Gholami, C. R. Chim., 2014, 17, 30-34.

19 Y.-H. Liu, J. Deng, J.-W. Gao and Zh.-H. Zhang, Adv. Synth. Catal., 2012, 354, 441-447.

20 G. M. Whitesides, Nature, 2006, 442, 368-373.

21 C. P. Park and D.-P. Kim, Angew. Chem., Int. Ed., 2010, 49, 6825-6829.

22 T. Rodrigues, P. Schneider and G. Schneider, Angew. Chem., Int. Ed., 2014, 53, 5750-5758.

23 Q. Deng, R. Shen, Z. Zhao, M. Yan and L. Zhang, Chem. Eng. J., 2015, 262, 1168-1174.

24 R. Porta, M. Benaglia and A. Puglisi, Org. Process Res. Dev., 2016, 20, 2-25.

25 N. J. W. Straathof, B. J. P. Tegelbeckers, V. Hessel, X. Wang and T. Noël, Chem. Sci., 2014, 5, 4768-4773.

26 D. Cantillo, O. de Frutos, J. A. Rincon, C. Mateos and C. O. Kappe, Org. Lett., 2014, 16, 896-899.

27 B. Gutmann, D. Cantillo and C. O. Kappe, Angew. Chem., Int. Ed., 2015, 54, 6688-6728.

28 A. Nagaki, K. Hirose, O. Tonomura, S. Taniguchi, T. Taga, Sh. Hasebe, N. Ishizuka and J. Yoshida, Org. Process Res. Dev., 2016, 20, 687-691.

29 P. Poechlauer, M. Vorbach, M. Kotthaus, S. Braune, R. Reintjens, F. Mascarello and G. Kwant, Micro Process Engineering, Wiley-VCH, Weinheim, 2009, vol. 3. 
30 M. G. A. Vieira, M. A. da Silva, L. O. dos Santos and M. M. Beppu, Eur. Polym. J., 2011, 47, 254-263.

31 G. Wypych, Handbook of Plasticizers, ChemTec Publishing, Toronto, 2nd edn, 2012.

32 M. Khodadadi Moghaddam and M. R. Gholami, Mater. Lett., 2006, 60, 715-719.

33 M. Yasunori and A. Masatomo, Jpn. Pat., JP 278840, 2001.
34 C. Xie, H. Li, L. Li, S. Yu and F. Liu, J. Hazard. Mater., 2008, 151, 847-850.

35 Z. Arabloo Narei, B. Shafiee and A. R. Khosropour, RSC Adv., 2015, 5, 20132-20137.

36 A. Schatz, M. Hager and O. Reiser, Adv. Funct. Mater., 2009, 19, 2109-2115. 